\title{
Determinación de la presencia de algunos compuestos químicos por métodos fitoquímicos colorimétricos en cinco especies forrajeras
}

\section{Determination of the presence of alkaloids, some chemical compounds by colorimetric phytochemicals methods in five forage species \\ Alarcón Julián ${ }^{1}$ y Navarro Cesar ${ }^{2}$ \\ ${ }^{1}$ Aspirante a Titulo de Médico Veterinario Zootecnista. ${ }^{2} \mathrm{MVZ}$. UNILLANOS \\ djulian.alarconh@gmail.com}

Recibido 15 Diciembre 2011, Aprobado 22 Febrero 2012

\section{RESUMEN}

Teniendo en cuenta la importancia que tiene para el Médico Veterinario y Zootecnista la nutrición animal y demás factores que constituyen el sistema salud - producción animal, y sabiendo que las propiedades nutricionales y antinutricionales están determinadas por la cantidad de planta ingerida y por el grado de presencia que tenga determinado metabolito en la misma, esta investigación se realizó en UNILLANOS, Villavicencio, y consideró como objetivo realizar un análisis fltoquímico, para determinar alcaloides, flavonoides, saponinas, esteroles y metilesteroles, en los árboles forrajeros: Bauhinia kalbreyeri (casco de vaca), Tithonia diversifolia (botón de oro), Brownea ariza (palo de cruz), Musaenda alicia (musaenda) y Megaskepasma erythrochlamys (capa roja brasileña); para en un futuro determinado hacer paralelos de investigación en cuanto a digestibilidad, degradabilidad, y demás características que determinan los beneficios de una especie forrajera. La determinación de alcaloides se realizo por el método rápido de Webb. En la prueba preliminar para la determinación de flavonoides, esteroles y metilesteroles se utilizó el método modificado de Wall y colaboradores; la primer prueba realizada fue la de Shinoda para determinar la presencia de flavonoides; la segunda prueba fue la de Libermann-Burchard para determinar la presencia de esteroles; y la última prueba fue la de Salkowshi. Para la determinación de saponinas se utilizo el método de Cain y colaboradores. Se determinó la presencia de alcaloides en Bauhinia kalbreyeri, Brownea ariza y 
Musaenda alicia, mientras que en Tithonia diversifolia y Megaskepasma erythrochlamys la prueba fue negativa. Igualmente se determinó la presencia de flavonoides en Brownea ariza, Bauhinia kalbreyeri, Musaenda alicia y Megaskepasma erythrochlamys, mientras que en Tithonia diversifolia la prueba fue negativa. Así mismo, se logro determinar la presencia de saponinas en Bauhinia kalbreyeri, Brownea ariza y Megaskepasma erythrochlamys, mientras que en Tithonia diversifolia y Musaenda alicia la prueba fue negativa. Por otro lado, no se logro determinar la presencia de esteroles y metilesteroles en las 5 especies estudiadas.

Palabras clave: Alcaloides, flavonoides, saponinas, esteroles.

\section{ABSTRACT}

Given the importance to the veterinarian and animal nutrition Zootechnist and other factors that constitute the system health-livestock, and knowing that the nutritional and antinutritional are determined by the amount of plant ingested and the degree of presence metabolite that has given it, this research was conducted in UNILLANOS, Villavicencio, and considered as target phytochemical analysis to determine alkaloids, flavonoids, saponins, sterols and methylsterols in fodder trees: Bauhinia kalbreyeri (casco de vaca), Tithonia diversifolia (botón de oro), Brownea ariza (palo de cruz), Musaenda alicia (musaenda) and Megaskepasma erythrochlamys (capa roja Brasileña), for in the future determined to do parallel research in terms of digestibility, degradability, and other characteristics determining the benefits of a forage species. The determination of alkaloids was carried out by the rapid method of Webb. In the preliminary test for the determination of flavonoids, sterols and methylsterols used the modified method of Wall et al, the first test performed was to Shinoda for the presence of flavonoids, the second test was to Libermann-Burchard to determine the presence of sterols, and the last test was to Salkowshi. For the determination of saponins was used the method of Cain et al. We determined the presence of alkaloids in Bauhinia kalbreyeri, Brownea ariza and Musaenda alicia, while the Tithonia diversifolia and Megaskepasma erythrochlamys test was negative. It was determined the presence of flavonoids 
in Brownea ariza, Bauhinia kalbreyeri, Musaenda alicia and Megaskepasma erythrochlamys, while the Tithonia diversifolia test was negative. Likewise, achieving the presence of saponins in Bauhinia kalbreyeri, Brownea ariza and Megaskepasma erythrochlamys, while the Tithonia diversifolia and Musaenda alicia test was negative. On the other hand, not achieving the presence of sterols and methylsterols in the 5 studied species.

Keywords: Alkaloids, flavonoids, saponins, sterols.

\section{INTRODUCCIÓN}

El término compuesto secundario engloba sustancias químicamente muy diversas y se establece como contraposición a los productos del metabolismo primario, que aparecen en el citoplasma de todas las células vegetales y cuyas diferencias entre plantas son únicamente de índole cuantitativo. En este sentido, se ha propuesto denominar a los compuestos secundarios sustancias ecológicamente eficaces, frente a los compuestos primarios que serían sustancias fisiológicamente eficaces (Strasburger et al., 1994). Los compuestos secundarios han sido durante mucho tiempo ignorados en los trabajos de nutrición. No obstante, su carácter claramente ventajoso para la planta y, en muchos casos, en obvio detrimento de los herbívoros, ha conducido a que actualmente se valore su incidencia en la producción agroganadera, especialmente en aquellos sistemas basados en el aprovechamiento de pastos (Ramos et al., 1998).

Se eligió la capa roja brasileña (Megaskepasma erythrochlamys), por existir datos del Laboratorio de Nutrición Animal de la Universidad de los Llanos en los cuales el porcentaje de proteína superaba el 20 hasta un 28\%, además de ser una especie que poco se ha estudiado nutricionalmente, al igual que Musaenda alicia.

Los alcaloides son, en su definición fundamental, compuestos heterocíclicos con nitrógeno y, como su nombre indica, sustancias generalmente de carácter básico, aunque existen muchas excepciones. El término abarca sustancias pertenecientes a grupos no relacionados entre sí, de las que se conocen más 
de 20.000 diferentes, entre cuyos precursores se encuentran varios aminoácidos. Según el estado químico del nitrógeno, se definen cuatro grupos: aminas secundarias y terciarias (alcaloides tipo), aminas cuaternarias y $\mathrm{N}$ óxidos (Ramos et al., 1998). Se presentan hasta en un 33\% de las plantas dicotiledóneas, estando, eso sí, ausentes de la mayoría de las monocotiledóneas (Cheeke y Palo, 1995). Los alcaloides son sustancias inodoras (Molyneux y Ralphs, 1992), no obstante, una característica de muchos grupos de alcaloides presentes en las plantas forrajeras es su sabor amargo, que posiblemente constituye la base para su identificación y consiguiente rechazo de la planta por los herbívoros (Harborne, 1993; Dupont et al., 1994). Sin embargo, los alcaloides derivados de la pirrolizidina, presentes en plantas ampliamente extendidas en los pastos, no poseen ninguna característica discernible en su palatabilidad por los herbívoros (Molyneux y Ralphs, 1992).

En las gramíneas dos grupos de alcaloides son los más importantes en cuanto a su efecto sobre el ganado doméstico: los derivados de la perlolina y perlolidina, con efectos tóxicos poco intensos y para los cuales, en ovejas, se ha indicado su rápida absorción y destrucción; y los derivados de la triptamina y gramina (Ramos et al., 1998). En experimentos con ovejas se ha visto que en la gramínea a niveles bajos $(0,01 \%)$ estimula el apetito, mientras que provoca el rechazo a niveles más altos (Harborne, 1993). La henificación favorece la disminución de la concentración de la gramínea (Tosi y Wittenberg, 1993). Sin embargo, los alcaloides derivados de la triptamina llevan a una intoxicación aguda que puede producir la muerte (Bush y Burton, 1994; Cheeke, 1995).

En las leguminosas se encuentran los alcaloides derivados del aminoácido lisina denominados quinolizidinas, distribuídos en varias tribus (Genisteae, Podalyrieae, y Sophoreae) de la subfamilia Papilionoideae. Dentro de las leguminosas herbáceas se halla el género Lupinus, los altramuces, que muestran una gran variabilidad intraespecífica en la presencia de los alcaloides (Múzquiz et al., 1993; Bush y Burton, 1994). Las variedades amargas y dulces de los altramuces se distinguirían, respectivamente, por su mayor o menor contenido en alcaloides (Múzquiz et al., 1993; Dupont et al., 1994). Las 
consecuencias del consumo de altramuces van desde la reducción de la ganancia media de peso y empeoramiento de los índices de transformación en pollos (Francesch et al., 1990), disminución de la ingestión en cerdos y vacas y reducción de la producción de leche en éstas (Mukisirat et al., 1995), alteraciones respiratorias, hasta la muerte del animal (Bush y Burton, 1994).

Los alcaloides del tipo de la pirrolizidina son un grupo de alcaloides que no son tóxicos por sí mismos en los mamíferos, sino que al ser metabolizados por las enzimas microsomiales en el hígado, se convierten en compuestos pirrólicos heterocíclicos que son las toxinas responsables de la mayoría de los efectos patológicos (Ramos et al., 1998). La molécula de pirrol se fija al hígado donde causa necrosis, o circula por el torrente sanguíneo causando daños en los pulmones; puede combinarse con moléculas de ADN ocasionando efectos mutagénicos y teratogénicos (Cheeke y Palo, 1995). Su presencia en plantas de amplia distribución en las zonas de pastoreo, hace que este tipo de alcaloides entre a formar parte con mucha frecuencia del alimento consumido por el ganado. Una característica de este tipo de alcaloides es su gran variabilidad en los efectos sobre las distintas especies animales. Así, por ejemplo, dentro de las especies de animales domésticos, caballos, cerdos, pollos y vacas son las más afectadas por la intoxicación debida a Senecio spp., mientras que ovejas, cabras y conejos son más resistentes (Smith, 1992).

Las saponinas se han empleado como detergentes naturales ya que son sustancias que rebajan la tensión superficial y producen espuma al contacto con el agua. Cuentan, además, con la capacidad de unirse al colesterol impidiendo su absorción (Gershenzon y Croteau, 1991). La mayoría de las plantas que contienen saponinas no tienen un sólo compuesto sino una mezcla compleja, con diferencias en la aglicona o en la longitud y composición de la cadena glicídica, lo que influye en sus propiedades (Oleszek, 1990; Massiot et al., 1991). Su toxicidad en los organismos animales no proviene tanto de su capacidad de lisis de los eritrocitos usada en las pruebas de determinación, sino de alterar la permeabilidad de la membrana celular, lo que sucede también en la pared intestinal (Gershenzon y Croteau, 1991; Önning et al., 1996). Un 
hecho derivado de lo anterior es la inhibición de las contracciones ruminales observadas por Klita et al., (1996) al administrar saponinas de raíz de alfalfa a dosis de un 4 y $8 \%$ de la ingestión de materia seca. Existe información contradictoria acerca de su efecto sobre la fermentación ruminal, digestibilidad, etc. (Klita et al., 1996; Önning et al. 1996). Al igual que los taninos, las saponinas podrían tener incidencia en la mejora de la eficiencia en la utilización del alimento en rumiantes, aumentando el flujo de proteína microbiana hacia el duodeno (Makkar et al., 1995; Klita et al., 1996), siendo su efecto más acusado cuando ambas sustancias se hayan presentes, que por separado (Makkar et al., 1995). Como consecuencia de daños hepáticos provocados por la deposición de cristales de saponinas esteroidales que se encuentran en algunas especies de gramíneas, puede ocurrir una fotosensibilización secundaria: el hígado dañado es incapaz de retirar de la circulación sanguínea un metabolito de la clorofila, la filoeritrina, que es un agente fotodinámico causante de fotofobia y dermatitis (Cheeke, 1995). Aunque se ha sospechado algún tipo de relación, no se ha encontrado asociación clara entre las saponinas presentes en la alfalfa y el timpanismo (Majak et al., 1995; Klita et al., 1996).

Flavonoide (del latín flavus, "amarillo") es el término genérico con que se identifica a una serie de metabolitos secundarios de las plantas que son sintetizados a partir de una molécula de fenilalanina y 3 de malonil-CoA, son una familia muy diversa de compuestos, aunque todos los productos finales se caracterizan por ser polifenólicos y solubles en agua. La composición y concentración de flavonoides es muy variable entre especies y en respuesta al ambiente. Un grupo importante de compuestos flavanoides en las plantas son los estrógenos vegetales, que se encuentran en forma de glucósidos, y cuya capacidad de modificar los procesos reproductivos proviene de su semejanza con el núcleo esteroídico de las hormonas femeninas (Ramos et al. 1998). Muchos otros compuestos secundarios afectan de una $u$ otra forma a la reproducción de los animales domésticos (James et al., 1992b; Porter y Thompson, 1992), pero los fitoestrógenos se encuentran en cantidades elevadas en especies vegetales que, como las pertenecientes a las 
leguminosas, son utilizadas intensamente como forraje. Los compuestos estrogénicos hallados en leguminosas pertenecen principalmente a tres grupos: isoflavanos, isoflavonas, presentes en el género Trifolium y prácticamente restringidas a las leguminosas, y cumestanos que se han estudiado en la alfalfa (Medicago sativa) y en Melilotus alba (Bush y Burton, 1994; Adams, 1995). Todas las isoflavonas sufren algún grado de transformación en el rumen, siendo el isoflavonoide formononetina, en realidad un proestrógeno, el único que se activa en el ecosistema ruminal al ser convertido en equol, mientras que las demás son degradadas a productos inactivos (Valderrábano, 1992; Harborne, 1993). Los procesos de henificación y ensilado pueden alterar la concentración de fitoestrógenos en el forraje (Bush y Burton, 1994; Adams, 1995). El mayor riesgo para los animales en pastoreo tiene lugar cuando las temperaturas son bajas, y el pasto está en período vegetativo, condiciones que aumentan la concentración de fitoestrógenos. Una prolongada exposición a pastos estrogénicos puede conducir a que la infertilidad sea permanente (Adams, 1995). Sin embargo, en los moruecos, parece que ni la fertilidad, ni la producción de esperma llegan a verse afectados (Valderrábano, 1992). En el ganado bovino los fitoestrógenos causan estros irregulares, ninfomanía, anestros y quistes ováricos, sin que hayan llegado a registrarse casos clínicos de infertilidad (Adams, 1995).

Los esteroles son esteroides con 27 a 29 átomos de carbono, formados por una cadena lateral de 8 ó más átomos de carbono (C) en el carbono 17 y un grupo alcohol o hidroxilo $(\mathrm{OH})$ en el carbono 3. Se caracterizan por poseer el ciclo perhidrofenantreno, el cual es un núcleo policíclico complejo que puede presentar enlaces dobles (insaturados) y ramificaciones. Estas sustancias se encuentran en abundancia en los organismos vivos, sobre todo en animales y en algunas algas rojas. Son solubles en los disolventes orgánicos, y poseen un elevado punto de fusión, destacan el colesterol y la vitamina D.

\section{MATERIALES Y MÉTODOS}

En las pruebas fotoquímicas preliminares se tomaron $100 \mathrm{~g}$ de planta seca y se dejo macerando en etanol durante 3 días. Este etanol macerado se filtro y se 
evaporo y el extracto que se obtuvo de esto, se utilizó para realizar las siguientes pruebas preliminares:

En la determinación de alcaloides, en primer lugar, se obtuvo el extracto etanolico, para ello una porción del residuo se disolvió en ácido clorhídrico diluido, se agitó y se filtró hasta que el filtrado quedó completamente transparente. El filtrado se ensayó con los reactivos para alcaloides: Mayer, Dragendorff, Wagner y Hager. Esta determinación se realizó por el método rápido de Webb (Pérez, 2006), el cual consistió en mezclar 5 g (Fotografía 1.) de material seco pulverizado con suficiente $\mathrm{HCl}$ al $1 \%$ para formar una suspensión y obtener $2 \mathrm{ml}$ de filtrado, la suspensión se vertió en un matraz Erlemeyer y se colocó en baño maría (Fotografía 2.) a $80^{\circ} \mathrm{C}$, la mezcla se calentó 4 horas y se agitó periódicamente. Después se retiró la suspensión, se dejó enfriar y se filtró (Fotografía 3.). Si el filtrado fue menor de $2 \mathrm{ml}$ se agregó suficiente $\mathrm{HCl}$ al $1 \%$ para ajustar el filtrado a $2 \mathrm{ml}$. Por separado se ensayaron alícuotas de $0.2 \mathrm{ml}$ del filtrado con volúmenes de $0.1 \mathrm{ml}$ de reactivo de alcaloides. Los resultados se registraron como: positivo si hubo formación de precipitado; y negativo si no hubo presencia de precipitado.

Para la preparación del reactivo de Mayer (Fotografía 4.) se disolvió $1.36 \mathrm{~g}$ de $\mathrm{HgCl}_{2}$ (cloruro de mercurio) en $60 \mathrm{ml}$ de agua y $5 \mathrm{~g}$ de $\mathrm{KI}$ (yoduro de potasio) en $10 \mathrm{ml}$ de agua, se juntaron las dos soluciones y se aforaron a $100 \mathrm{ml}$, vale la pena aclarar que el reactivo solo debe agregarse a soluciones previamente aciduladas con $\mathrm{HCl}$ (ácido clorhídrico) $0 \mathrm{H}_{2} \mathrm{SO}_{4}$ (ácido sulfúrico) diluido, la solución no debe tener acido acético o etanol. Solo debe agregarse unas cuantas gotas del reactivo porque algunos alcaloides son solubles en exceso de reactivo. Por otro lado, el reactivo de Hager, consiste en una solución saturada de pícrico en agua (Pérez, 2006).

En la prueba de Wagner se disuelven $1,27 \mathrm{~g}$ de yodo (resublimado) y $2 \mathrm{~g}$ de yoduro de potasio en $20 \mathrm{ml}$ de agua, la solución se afora a $100 \mathrm{ml}$ con agua destilada. La mayoría se soluciones aciduladas de alcaloides forman precipitados floculentos color marrón. En la prueba de Dragendorff se utilizan dos soluciones, en la solución A se disuelven $0,85 \mathrm{~g}$ de nitrato de bismuto 
pentahidratado con $20 \mathrm{ml}$ de ácido nítrico; en la solución B se disuelven $27,2 \mathrm{~g}$ de yoduro de potasio en $50 \mathrm{ml}$ de agua, se mezclan las 2 soluciones y se dejan en rasposo durante 24 horas, Se decanta la solución (para separar residuos de cristales de nitrito de potasio) y se afora a $100 \mathrm{ml}$. Se disuelven 1 a $2 \mathrm{mg}$ de la muestra en etanol y se colocan unas gotas en una placa de porcelana, luego se añaden unas gotas del reactivo de Dragendorff, la prueba es positiva para alcaloides al dar la placa precipitados de color rojo, naranja o marrón persistentes por 24 horas (Pérez, 2006).

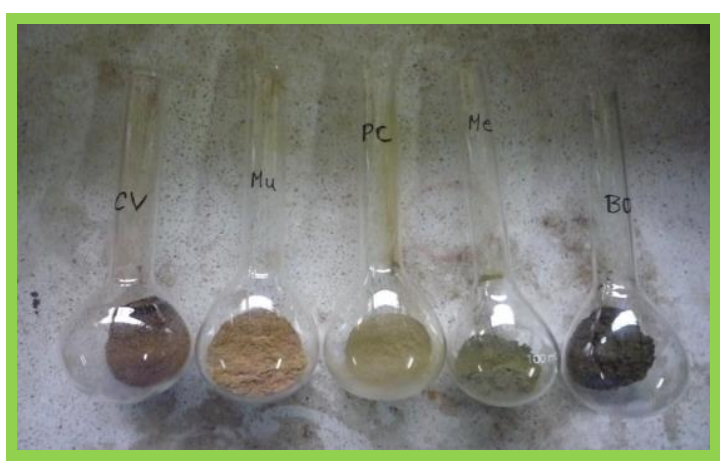

Fotografía 1. Muestras de los forrajes

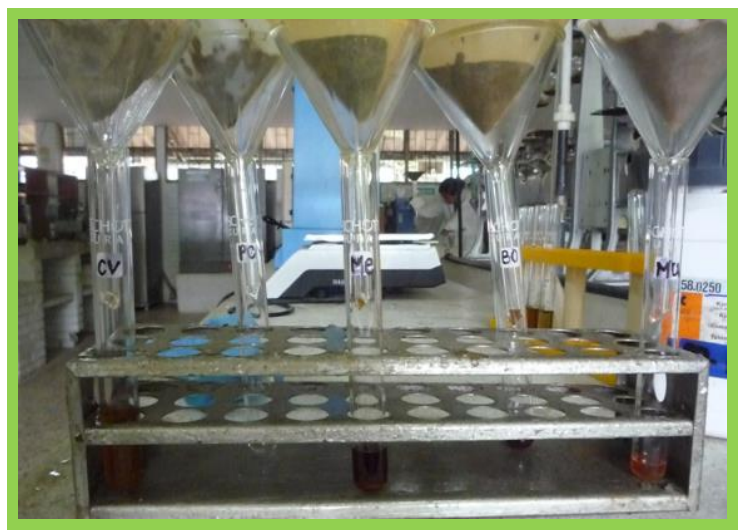

Fotografía 3. Extracto filtrado

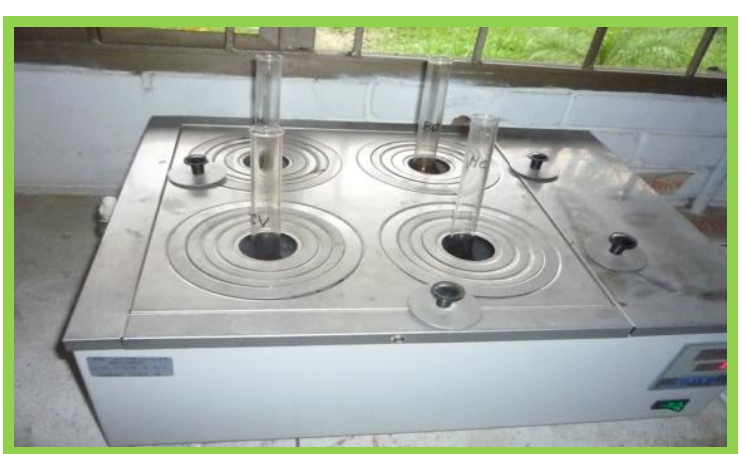

Fotografía 2. Baño maría

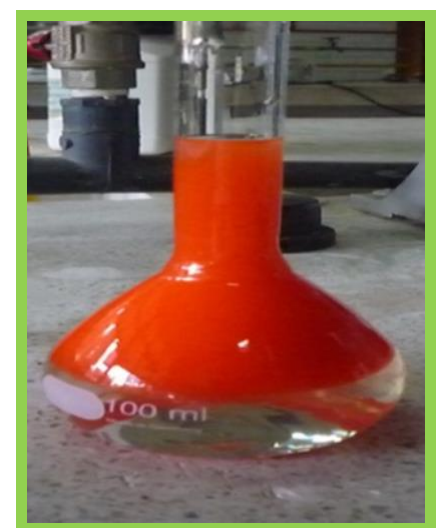

Fotografía 4. Reactivo de Mayer

En la prueba preliminar para la determinación de flavonoides, esteroles y metilesteroles se utilizó el método modificado de Wall y colaboradores, el cual consistió en tomar $20 \mathrm{~g}$ de planta pulverizada y secarla durante una hora en baño maría (Fotografía 6) con $100 \mathrm{ml}$ de etanol al 95\%. Se dejó enfriar la suspensión y se filtró. El material se lavó con 4 volúmenes de $50 \mathrm{ml}$ de etanol al $95 \%$. El filtrado y las soluciones lavadoras se juntaron y se evaporaron a $50^{\circ} \mathrm{C}$ y presión reducida hasta $50 \mathrm{ml}$, este concentrado se aforó a $100 \mathrm{ml}$ con 
agua, después se dividió en dos porciones, una para flavonoides y otra para esteroles y metilesteroles. La primer prueba realizada fue la de Shinoda (Pérez, 2006) para determinar la presencia de flavonoides, para la cual al filtrado en el tubo de ensayo se le agrega un trocito de magnesio, unas gotas de $\mathrm{HCl}$ concentrado y se considera positiva si vira de anaranjado a rojo o violeta; la segunda prueba fue la de Libermann-Burchard (Rossi et al., 2007) para determinar la presencia de esteroles, para ello se mezcló $1 \mathrm{ml}$ de anhídrido acético y uno de cloroformo, se enfrió la mezcla a $0^{\circ} \mathrm{C}$ (Fotografía 8) y se le añadió una gota de $\mathrm{H}_{2} \mathrm{SO}_{4}$. Esto se puso en contacto con el extracto de la planta. Se esperó el cambio de color dependiendo del tiempo de aparición azul (o minutos), verde (15 minutos), rojo (20 minutos), amarillo (60 minutos), si cambia a color amarillo después de 15 minutos parece corresponder a c-14 metilo. Hay que aclarar que en las pruebas para esteroles y metilesteroles no hay reacciones verdaderamente específicas puesto que hay otras sustancias que tienen características estructurales análogas; y la última prueba fue la de Salkowshi (Pérez, 2006), en la cual una pequeña cantidad de la muestra (1 mg en $1 \mathrm{ml}$ de cloroformo) se disolvió en $1 \mathrm{ml}$ de acido sulfúrico concentrado, se consideró positiva para flavonas y flavonoles si se observaron coloraciones amarillas, para chalcoinas coloraciones rojo-azul, y la presencia de quinonas con coloraciones rojo-purpura.

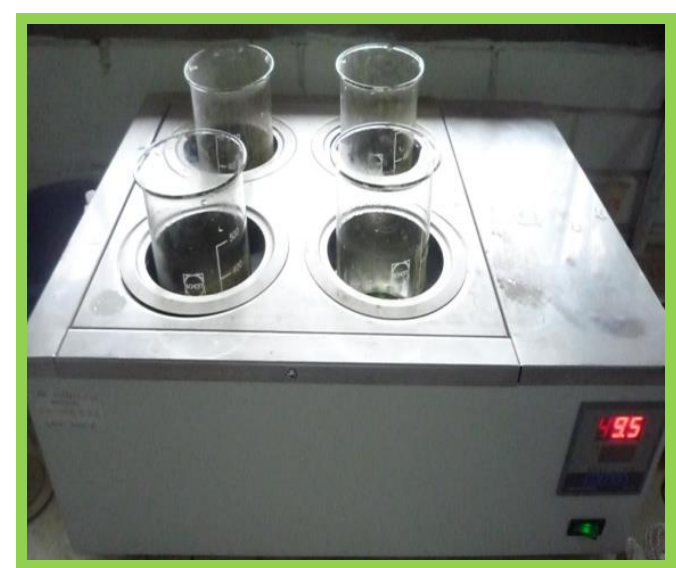

Fotografía 6. Baño maría

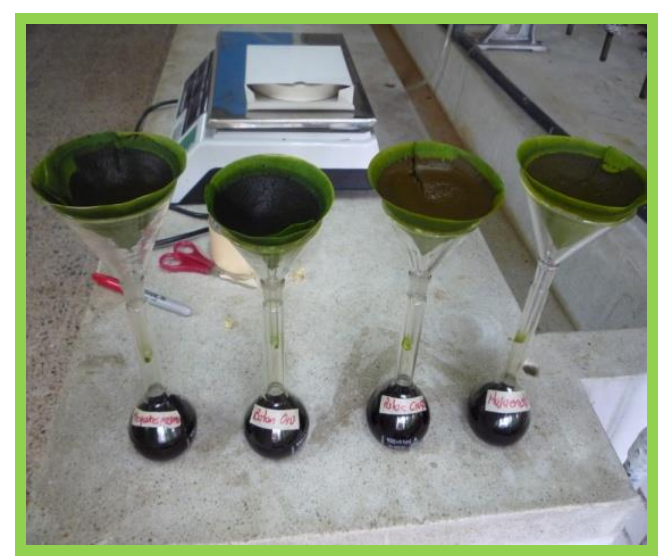

Fotografía 7. Filtrado

Para la determinación de saponinas se utilizo el método de Cain et al., (Pérez, 2006), para ello se tomaron $5 \mathrm{~g}$ de muestra, se le adicionó metanol, y mediante 
el equipo de soxhlet (Fotografía 9) se obtuvo un residuo rojo-café, semisólido el cual se utilizo para determinar la presencia de saponinas. Una porción de este residuo se disolvió con $\mathrm{H}_{2} \mathrm{O}$ caliente (en un tubo de ensayo) durante 15-30 minutos en baño maría, y luego se agitó vigorosamente durante 3-5 minutos. La formación de espuma con apariencia de panal de abejas, estable por unos 30 minutos, se considera prueba positiva.

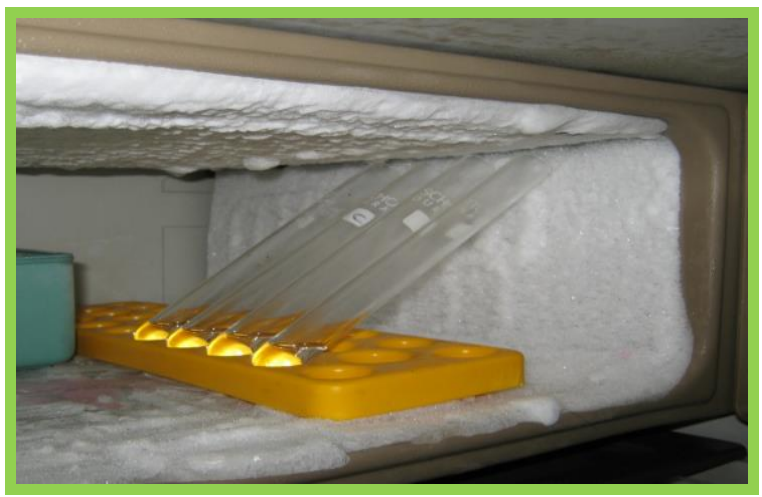

Fotografía 8. Enfriamiento a $0^{\circ} \mathrm{C}$.

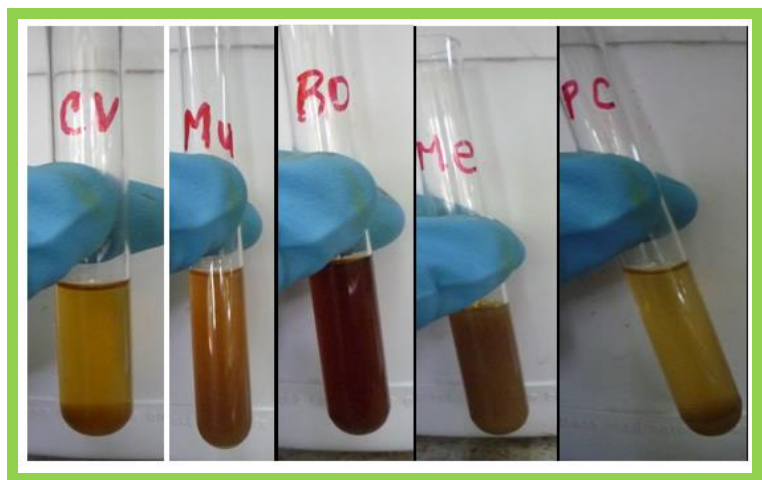

Fotografia 10. Prueba alcaloides

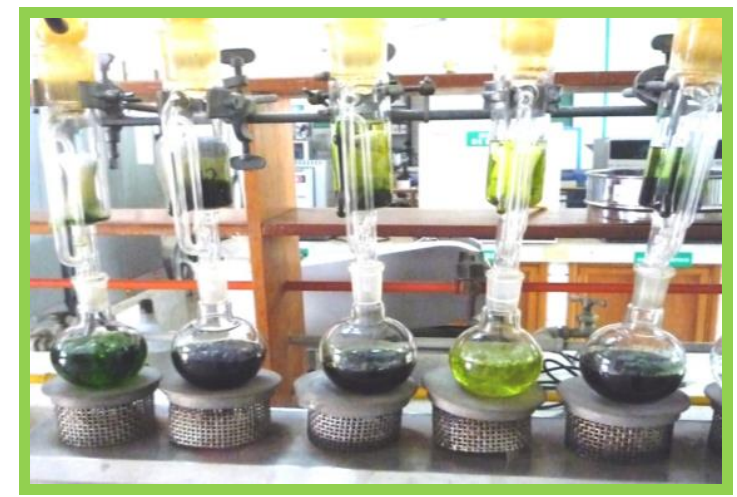

Fotografía 9. Equipo Soxhelt

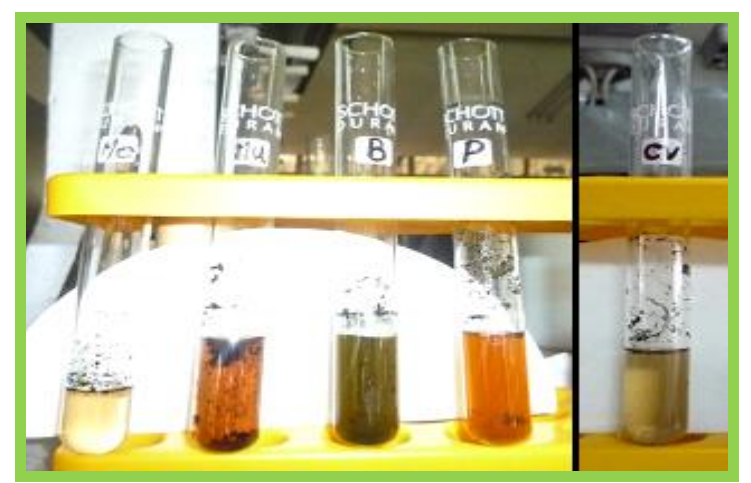

Fotografia 11. Prueba de Shinoda

\section{RESULTADOS Y DISCUSIÓN}

Los resultados de esta investigacion, coninciden con lo reportado por Gutierrez y Roa, (2003), quienes tambien determinaron la presencia de alcaloides en palo de cruz mediante metodos cualitativos. Se han encontrado alcaloides en un grupo de familias de plantas angiospermas y dicotiledóneas (Dominguez, 1973), no obstante no hay reportes recientes de las especies estudiadas, exceptuando el palo de cruz (Gutierez y Roa, 2003) 
Tabla 1. Resultados de la prueba de determinacion de alcaloides

\begin{tabular}{ll}
\hline \multicolumn{1}{c}{ ESPECIE } & \multicolumn{1}{c}{ RESULTADO } \\
\hline Bauhinia kalbreyeri & Positivo (+++) \\
Tithonia diversifolia & Positivo (+) \\
Brownea ariza & Negativo (-) \\
Musaenda alicia & Negativo (-) \\
Megaskepasma erythrochlamys & Positivo (++) \\
\hline Los resultados se registran como: & \\
Abundante (+++). Moderado (++). Escaso (+). Dudoso (+). Negativo (-). \\
\hline
\end{tabular}

Tabla 2. Resultados de la prueba de determinacion de flavonoides

\begin{tabular}{ll}
\hline \multicolumn{1}{c}{ ESPECIE } & \multicolumn{1}{c}{ RESULTADO } \\
\hline Bauhinia kalbreyeri & Positivo (+) \\
Tithonia diversifolia & Positivo (+++) \\
Brownea ariza & Negativo (-) \\
Musaenda alicia & Negativo (+++) \\
Megaskepasma erythrochlamys & Positivo (+) \\
\hline Los resultados se registran como: & \\
Abundante (++). Moderado (++). Escaso (+). Dudoso (+). Negativo (-). \\
\hline
\end{tabular}

En cuanto a la presencia de falvonoides, los resultados de esta investigacion tambien coninciden con lo reportado por Gutierrez y Roa (2003), quienes determinaron la presencia de dichos compuestos en palo de cruz mediante metodos cualitativos. De la misma manera hay conincidencia con lo reportado por Alberto y Solis, (2010) quienes tambien determinaron la presencia de flavonoides mediante la prueba de Shinoda en Megaskepasma erythrochlamys. Ortiz et al., (2009) también reportaron la presencia de flavonoides en Bauhinia kalbreyeri, además de determinar que dichos flavonoides tienen potencial antioxidante.

Los resultados de las pruebas para determinar la presencia de esteroles y metilesteroles en las 5 especies fueron negativos, por lo tanto, en la determinación de dichos compuestos hay diferencias con lo reportado por reportado por Gutierrez y Roa, (2003) quienes tambien determinaron la presencia de esteroides que contenian dos enlaces conjugados y/o presencia de triterpenos, mediante la prueba de Liebermann Burchard. Igualmente hay 
diferencias con lo reportado por Garcia et al., (2008) quienes tambien encontraron esteroles en Tithonia diversifolia.

Tabla 3. Resultados de la prueba de determinacion de saponinas

\begin{tabular}{ll}
\hline \multicolumn{1}{c}{ ESPECIE } & \multicolumn{1}{c}{ RESULTADO } \\
\hline Bauhinia kalbreyeri & Positivo (+++) \\
Tithonia diversifolia & Negativo (-) \\
Brownea ariza & Positivo (+++) \\
Musaenda alicia & Positivo (+) \\
Megaskepasma erythrochlamys & Positivo (-) \\
\hline Los resultados se registran como: & \\
Abundante (+++). Moderado (++). Escaso (+). Dudoso (+). Negativo (-). \\
\hline
\end{tabular}

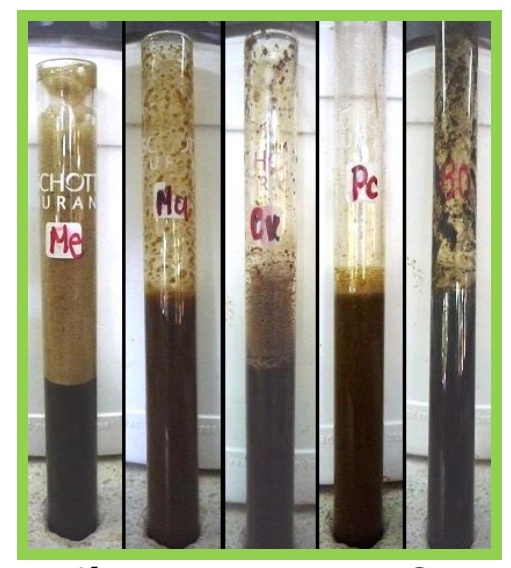

Fotografía 12. Prueba de Saponinas

En cuanto a la presencia de saponinas, se encontraron dichos compuestos en todas las especies arboreas estudiadas. Estos resultados tambien coninciden con lo reportado por Gutierrez y Roa, (2003) quienes determinaron la presencia saponinas en palo de cruz mediante metodos cualitativos.

Respecto a Tithonia diversifolia, en un análisis de metabolitos secundarios realizado por Rosales, (1992) no se encontraron fenoles ni taninos; mientras que Vargas, (1994) reportó un bajo contenido de fenoles y ausencia de saponinas. Mungarulire, (1993) encontró el compuesto citotóxico tagitinin; mientras que Dutta et al., (1993), además del compuesto tagitinin, encontraron hispidulin, a los cuales se le atribuyen efectos repelentes contra los insectos. 
La presencia de estos compuestos químicos en las especies estudiadas, amerita un análisis cuantitativo, que permita utilizar estas plantas en una forma más frecuente y confiable, aprovechando el potencial nutricional y la oportunidad que se presenta en las fincas del llano para su cultivo, además desde el punto de vista farmacológico se debe realizar un estudio orientado hacia la búsqueda de sus beneficios.

\section{CONCLUSIONES}

Se determinó la presencia de alcaloides en Bauhinia kalbreyeri (casco de vaca), Brownea ariza (palo de cruz) y Musaenda alicia (Musaenda), mientras que en Tithonia diversifolia (botón de oro) y Megaskepasma erythrochlamys (capa roja brasileña) la prueba fue negativa.

Se determinó la presencia de flavonoides en Bauhinia kalbreyeri (casco de vaca), Brownea ariza (palo de cruz), Musaenda alicia (Musaenda) y Megaskepasma erythrochlamys (capa roja brasileña), mientras que en Tithonia diversifolia (botón de oro) la prueba fue negativa.

No se logro determinar la presencia de esteroles y metilesteroles en las 5 especies estudiadas.

Se determinar la presencia de saponinas en Bauhinia kalbreyeri (casco de vaca), Brownea ariza (palo de cruz) y Megaskepasma erythrochlamys (capa roja brasileña), mientras que en Tithonia diversifolia (botón de oro) y Musaenda alicia (Musaenda) la prueba fue negativa.

\section{BIBLIOGRAFÍA}

1. Adams, N.R. Detection of effects of phytoestrogens on sheep and cattle. J. Anim. Sci., 73: 1509-1515. 1995.

2. Alberto, Y. G.; Solis, J. J. Extracción de colorantes naturales a partir de las inflorescencias de Ixora coccinea (IXORA), Bracteas de Alpinia purpurata (ginger rojo) y Megaskepasma erythrochlamys (camaroncillo) para su aplicación como indicadores acido-base en soluciones acuosas. Trabajo de graduación para optar al grado de licenciatura en química y farmacia. Universidad de el Salvador. San Salvador, El Salvador. 145 p. 2010.

3. Bush, L.; Burton H. Intrinsic chemical factors in forage quality. En: G.Fahey, Jr. (Ed.) Forage quality, evaluation and utilization. U.S.A. 1994. 
4. Cheeke, P. R. Endogenous toxins and mycotoxins in forage grasses and their effects on livestock. J. Anim. Sci., 73: 909-918. 1995.

5. Cheeke, P. R.; Palo, R. T. Plant toxins and mammalian herbivores: coevolutionary relationships and antinutritional effects. En: M. Journet, $E$. Grenet, M-H. Farce, M. Thériez, C. Demarquilly (Ed.) Recent developments in the Nutrition of Herbivores. Proceedings of the IV th International Symposium on the Nutrition of Herbivores pp: 437-456. INRA Editions, Paris. 1995.

6. Domínguez, X. Métodos de investigación fotoquímica. México, Editorial Limusa. 281 p. 1973

7. Dupont, M. S., Múzquiz, M., Estrella, I., Fenwick, G.R.; Price, K.R. Relationship between the Sensory Properties of Lupin Seed with Alkaloid and Tannin Content. Journal of Science Food Agriculture. 65: 95-100. 1994.

8. Dutta, P. et al. 1993. Insect feeding detergents from Tithonia diversifolia (Hemsl.) Gray. J. of Environmental Biology. 14 (1): 27.

9. Francesch, M., Pérez V. A., Esteve, E.; Brufau, J. Utilización de altramuz blanco (Lupinus albus) y altramuz azul (L. angustifolius) con contenido de alcaloides medio y alto en la alimentación del pollo de carne. Invest. Agr.: Prod. Sanid. Anim., 5: 91-107. 1990.

10. García, D.E., Medina, M. G., Cova, L.J., Torres, A., Soca, M., Pizzani, P., Baldizán, A.; Domínguez, C.E. Preferencia de vacunos por el follaje de doce especies con potencial para sistemas agrosilvopastoriles en el Estado Trujillo, Venezuela. Pastos y Forrajes. 31 (3): 255-270. 2008.

11. Gershenzon, J.; Croteau, R. Terpenoids. En: Rosenthal, G. A.; Berenbaum, M. R. (Ed.) Herbivores: Their Interactions with Secondary Plant Metabolites. p. 165-219. Academic Press, New York. 1991.

12. Gutiérrez, R.; Roa, M. L. Determinación de algunos compuestos químicos en cuatro plantas arbóreas forrajeras. Revista Colombiana de Ciencias Pecuarias. 16 (2): 155-161. 2003.

13. Harborne, J. B. Introduction to Ecological Biochemistry. Academic Press. London. 1993.

14. James, L. F., Panter, K. E., Nielsen, D. B.; Molyneux, R. J. The effect of natural toxins on reproduction in livestock. J. Anim. Sci., 70: 1573-1579. 1992.

15. Klita, P. T., Mathison, G. W., Fenton, T. W.; Hardin, R. T. Effects of alfalfa root saponins on digestive function in sheep. J. Anim. Sci., 74: 1144-1156. 1996.

16. Majak, W., Hall, J. W.; Mccaughey, W. P. Pasture management strategies for reducing the risk of legume bloat in cattle. J. Anim. Sci., 73: 1493-1498. 1995.

17. Makkar, H. P. S., Blümmel, M., Becker, K. In vitro effects of and interactions between tannins and saponins and fate of tannins in the rumen. J. Sci. Food Agric., 69: 481-493. 1995.

18. Molyneux, R. J.; Ralphs, M. H. Plant toxins and palatability to herbivores. J. Range Manage., 45: 13-18. 1992.

19. Mungarulire, J. Franz, C. H., Seitz, R.; Verlet, N. Some developments in the search for cytotoxic constituents from Rwandese medicinal plants. Acta Horticulturae. 333: 211. 1993. 
20. Múzquiz, M., Burbano, C., Cuadrado, C.; De La Cuadra, C. Determinación de factores antinutritivos termorresistentes en leguminosas. I: Alcaloides. Invest. Agr.: Prod. Prot. Veg., 8: 351-361. 1993.

21. Oleszek, W. Structural specificity of alfalfa (Medicago sativa) saponin haemolysis and its impact on two haemolysis-based quantification methods. J. Sci. Food Agric., 53: 477-485. 1990.

22. Önning, G., Wang, Q., Weström, B. R., Asp, N.; Karlsson, B. W. Influence of oat saponins on intestinal permeability in vitro and in vivo in the rat. Br. J. Nut., 76: 141-151. 1996.

23. Ortiz H. F., Sánchez, W. F. Méndez, J. A.; Murillo, E. P. Potencial antioxidante de hojas y corteza de Bauhinia kalbreyeri harms: contribución de sus flavonoides en esta actividad. Rev. Acad. Colomb. Cienc. 33: 127: 183-192. 2009.

24. Pérez L. E. Estudio fitoquímico biodirigido de las plantas con potencial actividad insecticida Trichilia havanensis y Croton ciliatoglanduliferus. Tesis de pregrado. Puebla, México. 70 p. 2006.

25. Porter, J. K.; Thompson, F. N. Effects of fescue toxicosis on reproduction in livestock. J. Anim. Sci. 70: 1594-1603. 1992.

26. Ramos, G.; Frutos P.; Giráldez F. J.; Mantecón A. R. Los compuestos secundarios de las plantas en la nutrición de los herbívoros. Revista Archivos de Zootecnia. 47: 597- 620. 1998.

27. Rosales, M. Nutritional value of Colombian fooder trees. Internal report. Fundación Centro para la Investigación en Sistemas Sostenibles de Producción Agropecuaria and Natural Resources Institute, United Kingdom. 50 p. 1992.

28. Rossi, C. A., De Leon, M., Gonzales, L.; Pereyra A. M. Presencia de metabolitos secundarios en el follaje de 10 leñosas de ramoneo en el bosque xerofítico del Cahco Arido argentino. Tropical and Subtropical Agroecosystems. 7 (2): 133-143. 2007.

29. Smith, G. S. Toxification and detoxification of plant compounds by ruminants: an overview. J. Range Manage. 45: 25-30. 1992.

30. Strasburger, E., Noll, F., Schenk, H.; Schimper, A. F. W. Tratado de Botánica. Ed. Marín. Barcelona. 1994.

31. Tosi, H. R.; Wittenberg, K. M. Harvest alternatives to reduce the alkaloid content of reed canarygrass forage. Can. J. Anim. Sci., 73: 373-380. 1993.

32. Valderrábano, J. Alteraciones reproductivas asociadas al consumo de fitoestrógenos. Invest. Agr.: Prod. Sanid. Anim., 7: 115-124. 1992.

33. Vargas, J. E. Caracterización de recursos forrajeros disponibles en tres agroecosistemas del Valle del Cauca. En: Memorias II Seminario Internacional Desarrollo sostenible de Sistemas Agrarios. Maestría en Sistemas Sostenibles de Producción Animal en los Trópicos. Cali, Colombia. p. 135. 1994. 